\title{
HIV-1 and Syphilis Prevalence Among Local Chinese and Cross-border Migrant Female Sex Workers in Sino-Vietnam Border Cities in Guangxi, China: a Nine-year Consecutive Cross-sectional Study
}

\section{Bingyu Liang}

Guangxi Medical University

Fei Zhang

Guangxi Medical University https://orcid.org/0000-0002-3789-0321

Dongmei Wei

Chongzuo Centers for Disease Control and Prevention

Peidong Zhang

Fangchenggang Center for Disease Control and Prevention

Guangwu Wei

Chongzuo Center for Disease Control and Prevention

Shide Mo

Fangchenggang Center for Disease Control and Prevention

Aidan Nong

Chongzuo Center for Disease Control and Prevention

Yanyun Ou

Chongzuo Center for Disease Control and Prevention

Zhenxian Wu

Chongzuo Center for Disease Control and Prevention

Hai Xie

Fangchenggang Center for Disease Control and Prevention

Xu Liang

Baise Center for Disease Control and Prevention

Jiegang Huang

Guangxi Medical University

Junjun Jiang

Guangxi Medical University

Jie Liu

Guangxi Medical University

Yanyan Liao

Guangxi Medical University

Deping Liu

Chongzuo Center for Disease Control and Prevention

Hao Liang

Guangxi Medical University

Li Ye ( $\nabla$ yeli@gxmu.edu.cn )

Guangxi Medical University https://orcid.org/0000-0001-7688-4867

\section{Research}

Keywords: HIV infection, syphilis infection, female sex workers, risk behaviours, trends, Sino-Vietnam border

Posted Date: October 6th, 2021

DOI: https://doi.org/10.21203/rs.3.rs-400830/v2

License: (c) (i) This work is licensed under a Creative Commons Attribution 4.0 International License. Read Full License 


\section{Abstract}

Background To analyze the trends of HIV-1 and syphilis prevalence as well as associated factors among local Chinese female sex workers (LCFSWs) and cross-border migrant female sex workers (CMFSWs) in Sino-Vietnam border cities in Guangxi, China.

Methods A consecutive cross-sectional study was conducted from 2010 to 2018 in three border cities in Guangxi. The survey was conducted through face-toface interview with a structured questionnaire. HIV-1 and syphilis infections were tested simultaneously. Cochran-Armitage analysis was used to assess the trends of HIV-1, syphilis and HCV prevalence, as well as the trend of risk behaviors, HIV/AIDS knowledge and prevention services. Multivariate logistics regression analysis was used to identify factors associated with HIV-1 and syphilis infection.

Results Totally, 19873 LCFSWs and 1723 CMFSWs were enrolled. The overall prevalence of HIV-1 and syphilis infection was $0.91 \%$ and $5.14 \%$, respectively. The HIV-1 prevalence among CMFSWs was higher than that among LCFSWs, without a declined trend. However, a downward trend was observed among LCFSWs. Overall, the main associated factors of HIV-1 infection among CMFSWs shifted from age $>35$ years old, staying in current residence for $>12$ months, self-reporting sexually transmitted infections (STIs) in the last year during 2010-2015 to inconsistent condoms use in commercial sex in last month, not receiving peer education and self-reporting STIs in the last year during 2016-2018. The main correlated factors of syphilis infection among CMFSWs changed from history of illicit drug use, staying in current residence for more than 12 months, self-reporting of STls and inconsistent condoms use in commercial sex in the past month during 2010-2015 to working in low-tier venues, self-reporting of STIs in the last year, and sexing with commercial male clients who used aphrodisiac drugs during 2016-2018. For LCFSWs, the relative strong associated factors of HIV-1 infection shifted from receiving less than 6 years of education, illicit drugs use, and self-reporting STIs in the last year during 2010-2015 to sexing with commercial male clients who use aphrodisiac drugs and self-reporting of STIs during 2016-2018, while the main factors of syphilis infection changed from self-reporting STIs in the last year, history of illicit drug use and receiving less than 9 years of education during 2010-2015 to self-reporting of STIs in the last year, sexing with commercial male clients who use aphrodisiac drugs, and history of illicit drugs use during 2016-2018.

Conclusions The relatively higher prevalence of HIV-1 infection and frequent risk behaviours among CMFSWs in border cities in Guangxi, indicating that CMFSWs is the high-risk population for HIV-1 infection and transmission in the region, and is the key population of HIV/AIDS prevention and control. Meanwhile, the change of HIV infection-associated risk factors over time suggests that HIV intervention strategies need to be changed accordingly.

\section{Background}

The HIV/AIDS epidemic is a significant public health problem in China, especially in southern areas, including Guangxi province[1]. By the end of 2018, there more than 80,000 people living with HIV/AIDS in Guangxi, accounting for one-tenth of the total number of cases in China[2]. Since 2006, the predominant route of HIV-1 transmission in Guangxi has gradually shifted from injection of drug use to heterosexual contact[3]. Moreover, the proportion of new HIV-1 cases attributed to heterosexual contact increased from less than $10 \%$ to over $90 \%[3]$. The increasing prevalence of HIV-1, especially via heterosexual contact, is alarming as it raises the potential for a more diffuse and generalized epidemic. Female sex workers (FSWs) who engage in sexual services in exchange for money or goods are at high risk of contracting HIV-1 and other sexually transmitted infections (STIs) such as syphilis[4-6], facilitating the current heterosexually-driven HIV/STIs epidemics, and are considered as a bridge group for the transmission of HIV/STIs from high-risk groups to the general population[7].

Although the prevalence of HIV-1 among FSWs in China has been decreasing in recent years[8], Guangxi, located in the southwest of China, is still the region with a high HIV-1 burden[9]. From 2010 to 2012, the prevalence of HIV-1 and syphilis among FSWs in Guangxi were 1.0\% and 6.7\%, respectively[10], with the former higher than the national level and the latter was similar to national average[11]. A cross-sectional study of four Chinese Provinces revealed that FSWs from Guangxi were nearly eight times more likely to be HIV-1 positive than those from other regions[12]. Meanwhile, disparities in working venues also contribute to the differences in HIV-1 and syphilis prevalence among FSWs in Guangxi: the prevalence of HIV-1 among high, middle and low-tier FSWs was reported to be $0.5 \%, 0.5 \%$ and $1.9 \%$, while the prevalence of syphilis was $3.2 \%, 4.5 \%$ and $10.5 \%$, respectively[10]. Low education level, low knowledge of STIS/HIV, low-tier working venues and no condom use during last sexual intercourse were correlated with STIs and HIV-1 infection among FSWs in Guangxi[13].

Additionally, Guangxi shares a 1020-kilometre borderline with Vietnam, and is in close proximity to the "Golden Triangle", an area known as the entry point for drug trafficking routes and cross-border sex worker migration. Under China's Belt and Road Initiative, the commercial relationship between Guangxi and Vietnam is growing rapidly in recent years. This has led to a relatively high number of commercial sex and illicit drug use behaviours in border cities within Guangxi. Study has shown that the proportion of FSWs using condoms in cross-border migrants was only 39.5\%, far lower than the proportion of local Chinese female sex workers (LCFSWs) using condoms in border counties of Guangxi (88.4\%)[14]. Higher HIV-1 prevalence was observed among cross-border migrant female sex workers (CMFSWs) than LCFSWs in Guangxi, ranging from 3.2\%[15] to 8.2\%[9]. The overall HIV-1 prevalence in FSWs in five border Provinces in Vietnam also reached 4.5\%[16]. An early age of sexual debut, low-tier venues and the more years of experience as sex worker were significant risk factors for HIV-1 infection in border areas[15, 17].

Much previous work had focused on HIV/STIs prevalence and their associated factors among FSWs, while few studies have used a consecutive crosssectional study to longitudinally reveal the trends of HIV-1 prevalence and related behaviours. In addition, male clients without steady commercial sexual partner have been shown to be at higher risk of HIV-1 and syphilis infection, which were affected by a variety of characteristics of commercial sex[18]. Previous study also demonstrated that aphrodisiac use associated with HIV infection among elderly male clients at low-cost commercial sex venues[19]. However, few studies have examined whether the demographic or sexual behaviors of male clients have an impact on STIs or HIV-1 infection in FSWs. We hypothesize that the acquisition of syphilis and HIV-1 among FSWs is influenced by their sexual behaviors and demographic and their male clients' sexual 
behaviors and demographic, such as inconsistent condom use, aphrodisiac drugs use and age. To date, no comprehensive assessments of trends in the burden of HIV-1 and other STIs among FSWs in the Sino-Vietnam border areas are available.

This serial cross-sectional study aimed to explore the trends of HIV-1 and syphilis prevalence and risk behaviours over nine years, evaluate the HIV-1 and syphilis burden in Sino-Vietnam border areas in Guangxi and identify associated factors of HIV/STIs in LCFSWs and CMFSWs during 2010-2015 and 20162018 two periods, respectively.

\section{Methods}

\section{Study population and data collection}

This consecutive cross-sectional study was conducted in three Sino-Vietnam cities (Chongzuo, Fangchenggang, and Baise city) in Guangxi. From April 2010 to June 2018, a total of 21,596 participants were recruited, who were further included in this study with inclusion criteria: who were at least 16 years of age, provided sexual services in exchange for money for more than 4 times per month in the past six months. Venue-based sampling was used in recruiting participants. A 30 mins' Face-to-face interview was conducted at a separate room in the FSWs' working venue by female trained staff from the Chinese local Center for Disease Control and Prevention (CDC). All the interviewed staffs were fluent in both Mandarin and Vietnamese. Participants of Chinese nationality were defined as local Chinese FSWs (LCFSWs), otherwise, they were defined as cross-border migrant FSWs (CMFSWs). LCFSWs and CMFSWs were interviewed in Mandarin and Vietnamese, respectively. Venous blood samples were collected by trained professional physicians. Detailed descriptions of sampling and recruitment methods can be found elsewhere[20].

\section{Demographic and behavioural variables measurement}

The participants first provided relevant demographic information, including working venues, age, marital status, educational background, months of stay in current residence. The participants were categorized into low-tiers and high-tiers based on their working venues[8]. The low-tiers include hair salon, barbershop, massage parlour, foot-bath shop, guesthouse, roadside restaurant, street and other outdoor public area; and the high-tiers include karaoke bar, hotel and other establishment accessible only to those of higher socioeconomic status. Variables regarding condom use in last commercial sex, condom use in commercial sex in the past month, history of illicit drugs use were also collected. Remarkably, a more detailed questionnaire was designed and used to further elucidate whether characteristics of their male clients or sexual partners had an impact on HIV-1 and syphilis infection from 2016 to 2018 . Therefore, information on months of stay in the same venue, years of experience as FSW, age at commercial sexual debut, number of male clients in the past month, sex with regular commercial sexual partners in the past month, presence of steady non-commercial sexual partners, age of male clients, and commercial sex with male clients who used aphrodisiac drugs were also taken into account during 2016-2018.

\section{HIV/AIDS knowledge and intervention services}

HIV/AIDS-related knowledge was assessed with eight basic questions regarding HIV transmission routes, treatment and preventive measures. Eight HIV/AIDSrelated questions were as following:(1) Is AIDS a serious infectious disease with no cure? (2) Is sexual transmission the main mode of HIV transmission in China? (3) Can a person with HIV be recognized by appearance? (4) Does it increase the risk of HIV infection if someone has a sexually transmitted disease? (5) Can condom use reduce the risk of infecting and transmitting HIV? (6) Does new type of drug use (such as methamphetamine, ecstasy, ketamine, etc.) increase the risk of HIV infection? (7) Should someone actively seek HIV testing and counseling after high risk behaviors (needle sharing, drug use, unsafe sex, etc.)? (8) Is there legal liability for intentionally spreading HIV? The options for those question were "Yes", "No", I don't know". Those who had a cumulative score of 6 or above were considered as having accurate HIV-/AIDS-related knowledge[21]. In addition, participants reported whether they had ever receiving any HIV intervention services, including free condom distribution programmes, methadone maintenance therapy or needle exchange programmes and peer education programmes, in the past year.

\section{Self-reporting of STIs and tests for HIV, syphilis and HCV}

Participants reported whether they had a history of gonorrhoea, syphilis, condyloma acuminata, genital herpes, or any other STIs in the last year. Briefly, blood specimens were tested for antibodies against HIV-1 and syphilis. HIV-1 antibody testing was performed according to standardised operating procedures based on the manufacturer's instructions. Any specimen that screened positive by Enzyme-linked immunosorbent assay (ELISA) (Wantai Biological Pharmaceutical Co., Beijing, China) was confirmed by western blot (WB) assay (HIV Blot 2.2 WB; Genelabs Diagnostic, Singapore). For syphilis, rapid plasma reagin (RPR) (Rongsheng Biotechnical Company, Shanghai, China) was used as a screening test, and positive samples were confirmed by Treponema pallidum particle agglutination (TPPA, Serodia; Fujirebio, Fuji, Japan). Subjects with positive results for both RPR and TPPA were considered to have a current syphilis infection. Subjects with positive results for both RPR and TPPA were considered to have a current syphilis infection. HCV antibody testing was done using an IgG-based ELISA (Wantai Biological Pharmaceutical Co., Beijing, China).

\section{Statistical analysis}

The statistical analysis was completed using SPSS Statistics 26.0 (SPSS Inc, Chicago, IL, USA). Chi-square tests were used to compare the differences between LCFSWs and CMFSWs. In order to show the trend of infections and behavior variables more clearly, cochran-Armitage trend analysis was used to assess the trends of HIV-1 and syphilis prevalence, risk behaviours and the FSWs exposure to the HIV/AIDS-related interventions. HIV-1, syphilis infection and related risk behaviours and interventions were treated as dependent variables, while time was treated as an independent variable (data not showed).

Due to low prevalence of HIV and syphilis in this population, the multivariable model did not converage for all covariates. First, we performed bivariable analyses for each independent variable on HIV and syphilis and odds ratios (OR) and 95\% Cls were computed. Then all variables associated whith HIV or syphilis at a $\mathrm{P}<0.05$ in the bivariable analyses were tested for multicollinearity. The tolerances of all the independent variables were all greater than 0.1 , and 
the variance inflation factor (VIF) were all less than 5 , indicating low collinearity. Next, we conducted Correlation matrix of all the independent variables with low collinearity. The correlation coefficient less than 0.7 indicates low correlation. Finally,we constructed the multivariable model by including all the determinants of interest described in the analytical framework and individual level determinants with low collinearity and low correlation with other variables, and adusted odds ratio (AOR) and $95 \%$ confidence interval $(\mathrm{Cl})$ were calculated. $p$-value $\leq 0.05$ was considered statistically significant.

\section{Results}

\section{Demographic characteristics}

A total of 21,596 FSWs were recruited in this study over nine years. The demographic characteristics were shown in Table 1. Among all the participants, 19,873 (92.0\%) were LCFSWs and 1,723 (8.0\%) were CMFSWs. It is worth noting that all of the CMFSWs in our study were from Vietnam. Additionally, results showed that CMFSWs were more likely to be unmarried, divorced or widowed, receiving less than 6 years of education, working in low-tier venues, reporting a shorter time of staying in current residence, a shorter length time as a commercial sex worker and a younger age at commercial sexual debut than LCFSWs.

Table 1

Demographic characteristics of local Chinese and cross-border migrant FSWs (\%)

\begin{tabular}{|c|c|c|c|c|c|c|c|c|}
\hline \multirow[t]{3}{*}{ Variables } & 2010 & 2011 & 2012 & 2013 & 2014 & 2015 & 2016 & 2017 \\
\hline & $N=1680$ & $N=2366$ & $N=2461$ & $N=3350$ & $N=3197$ & $N=3239$ & $N=1061$ & $N=2185$ \\
\hline & CM & CM & $\mathrm{CM}$ & $\mathrm{CM}$ & $\mathrm{CM}$ & $\mathrm{CM}$ & $\mathrm{CM}$ & LC \\
\hline
\end{tabular}

\section{Working venues}

\begin{tabular}{|c|c|c|c|c|c|c|c|c|c|c|c|c|c|c|c|c|}
\hline Low-tier & 89.1 & 78.6 & 93.9 & 83.1 & 92.5 & 92.3 & 89.7 & 96.5 & 90.2 & 99.3 & 89.8 & 94.7 & 52.3 & 43.9 & 43.4 & 80.6 \\
\hline High-tier & 10.9 & 21.4 & 6.1 & 16.9 & 7.5 & 7.7 & 10.3 & 3.5 & 9.8 & 0.7 & 10.2 & 5.3 & 47.7 & 56.1 & 56.6 & 19.4 \\
\hline \multicolumn{17}{|l|}{ Age } \\
\hline$>35$ years old & 15.3 & 21.4 & 16.3 & 16.7 & 19.7 & 16.2 & 26.2 & 14.5 & 27.0 & 27.7 & 25.5 & 22.5 & 46.2 & 28.8 & 37.6 & 31.8 \\
\hline$\leq 35$ years old & 84.7 & 78.6 & 83.7 & 83.3 & 80.3 & 83.8 & 73.8 & 85.5 & 73.0 & 72.3 & 74.5 & 77.5 & 53.8 & 71.2 & 62.4 & 68.2 \\
\hline \multicolumn{17}{|l|}{ Marital status } \\
\hline Married/cohabiting & 52.0 & 35.7 & 56.2 & 43.9 & 60.7 & 43.8 & 60.9 & 47.3 & 62.6 & 59.5 & 65.5 & 55.7 & 64.9 & 66.9 & 64.4 & 69.9 \\
\hline Unmarried/divorced/widowed & 48.0 & 64.3 & 43.8 & 56.1 & 39.3 & 56.2 & 39.1 & 52.7 & 37.4 & 40.5 & 34.5 & 44.3 & 35.1 & 33.1 & 35.6 & 30.1 \\
\hline \multicolumn{17}{|l|}{ Years of education } \\
\hline$<6$ years & 33.3 & 50.0 & 28.7 & 48.5 & 29.4 & 47.7 & 29.7 & 35.3 & 28.5 & 49.3 & 34.8 & 39.7 & 48.6 & 14.4 & 33.8 & 46.8 \\
\hline $6-9$ years & 59.1 & 50.0 & 61.7 & 40.9 & 63.8 & 49.2 & 61.3 & 53.4 & 65.8 & 40.2 & 58.4 & 37.0 & 46.7 & 47.5 & 58.9 & 47.2 \\
\hline$>9$ years & 7.6 & 0.0 & 9.6 & 10.6 & 6.8 & 3.1 & 9.0 & 11.3 & 5.7 & 10.5 & 6.8 & 23.3 & 4.7 & 38.1 & 7.3 & 6.0 \\
\hline
\end{tabular}

Months of staying in current residence

\begin{tabular}{|c|c|c|c|c|c|c|c|c|c|c|c|c|c|c|c|c|}
\hline$>12$ months & 41.2 & 61.9 & 37.1 & 33.3 & 48.2 & 50.8 & 41.4 & 27.6 & 42.1 & 34.1 & 37.5 & 23.7 & 50.2 & 19.4 & 46.9 & 42.8 \\
\hline $6 \sim$ & 17.0 & 14.3 & 18.8 & 12.1 & 18.9 & 18.0 & 17.9 & 14.6 & 21.3 & 16.6 & 20.8 & 11.8 & 10.5 & 7.9 & 21.5 & 15.4 \\
\hline$<6$ months & 41.8 & 23.8 & 44.1 & 54.6 & 32.9 & 31.2 & 40.7 & 57.8 & 36.6 & 49.3 & 41.7 & 64.5 & 39.3 & 72.7 & 31.6 & 41.8 \\
\hline
\end{tabular}

Age of commercial sexual debut

\begin{tabular}{|c|c|c|c|c|c|c|c|c|c|c|c|c|c|c|c|c|}
\hline$\geq 18$ years old & - & - & - & - & - & - & - & - & - & - & - & - & 98.4 & 92.1 & 98.6 & 98.0 \\
\hline$<18$ years old & - & - & - & - & - & - & - & - & - & - & - & - & 1.6 & 7.9 & 1.4 & 2.0 \\
\hline
\end{tabular}

\section{Years of experience as FSW}

\begin{tabular}{|c|c|c|c|c|c|c|c|c|c|c|c|c|c|c|c|c|}
\hline$>5$ years & - & - & - & - & - & - & - & - & - & - & - & - & 37.2 & 7.9 & 31.5 & 16.1 \\
\hline$\leq 5$ years & - & - & - & - & - & - & - & - & - & - & - & - & 62.8 & 92.1 & 68.5 & 83.9 \\
\hline \multicolumn{17}{|c|}{ Months of staying in the same venue } \\
\hline$>12$ months & - & - & - & - & - & - & - & - & - & - & - & - & 30.5 & 33.1 & 18.9 & 15.4 \\
\hline$\leq 12$ months & - & - & - & - & - & - & - & - & - & - & - & - & 69.5 & 66.9 & 81.1 & 84.6 \\
\hline
\end{tabular}




\section{Prevalence of HIV-1, syphilis and HCV infection}

The overall prevalence of HIV-1 and syphilis infection among all the participants was $0.91 \%(197 / 21596)$ and 5.14\% (1109/21596), respectively, and remained relatively stable during the study period. However, as shown in Fig. 1, the HIV-1 prevalence among LCFSWs significantly decreased from 2010 to 2018 (OR = 0.922, 95\% Cl: 0.859-0.989; $p$ for trend $=0.024$ ), but this trend was not found among CMFSWs. Furthermore, higher HIV-1 and HCV prevalence was observed among CMFSWs than LCFSWs (HIV-1: $3.13 \%$ versus $0.72 \%, p<0.001$; HCV: $2.09 \%$ versus $1.05 \%, p<0.001$ ), though HCV prevalence significantly decreased among CMFSWs from 2010 to 2018 (OR $=0.845,95 \% \mathrm{Cl}: 0.726-0.983 ; p$ for trend $=0.029)$. There was no statistical difference in the prevalence of syphilis infection between LCFSWs and CMFSWs $(5.15 \%$ versus $4.93 \%, p=0.838)$, and there was no significant change in the trend of syphilis infection of the two groups.

\section{Behavioural characteristics and self-reporting of STIs}

As shown in Table 2, compared to LCFSWs, CMFSWs were more likely to use illicit drugs, sex with regular commercial sexual partners in the past month, but less likely to use condoms in last commercial sex and in the last month, have steady non-paying sexual partners and sex with commercial male clients who used aphrodisiac drugs. The proportion of participants who insisted on using condoms in last commercial sex and in last month were both gradually increased among LCFSWs (OR = 1.364, 95\% Cl: $1.315-1.415 ; \mathrm{OR}=1.344,95 \% \mathrm{Cl}: 1.319-1.369)$ and CMFSWs $(\mathrm{OR}=1.242,95 \% \mathrm{Cl}: 1.122-1.375 ; \mathrm{OR}=1.123$, $95 \% \mathrm{Cl}: 1.061-1.188)$. History of illicit drugs use was gradually increased among LCFSWs (OR $=1.075,95 \%$ Cl: $1.020-1.134)$, while it was gradually decreased among CMFSWs (OR $=0.771,95 \% \mathrm{Cl}: 0.666-0.892)$. Downward trends were also found in the number of male clients in the past month $(\mathrm{OR}=0.915,95 \% \mathrm{Cl}$ : $0.847-0.987)$, sexing with regular commercial sexual partners in the past month (OR $=0.892,95 \% \mathrm{Cl}: 0.830-0.959)$ and having steady non-paying sexual partners $(\mathrm{OR}=0.837,95 \% \mathrm{Cl}: 0.752-0.932)$ among LCFSWs. CMFSWs also reported a decreasing trend of sexing with regular commercial sexual partners in the past month in the study period (OR $=0.375,95 \% \mathrm{Cl}: 0.301-0.467)$. The difference of Self-reporting of STIs in the last year between LCFSWs and CMFSWs was not statistically significant, but gradually increased among the LCFSWs $(\mathrm{OR}=1.052,95 \% \mathrm{Cl}: 1.021-1.084)$. 
Table 2

Behavioural, HIV/AIDS knowledge and prevention services of local Chinese and cross-border migrant FSWs from 2010 to 2018 (\%)

\begin{tabular}{|c|c|c|c|c|c|c|c|c|c|c|c|c|c|c|}
\hline \multirow[t]{2}{*}{ Variables } & & \multirow{2}{*}{$\begin{array}{l}2010 \\
N= \\
1680\end{array}$} & \multirow{2}{*}{$\begin{array}{l}2011 \\
N= \\
2366\end{array}$} & \multirow{2}{*}{$\begin{array}{l}2012 \\
N= \\
2461\end{array}$} & \multirow{2}{*}{$\begin{array}{l}2013 \\
N= \\
3350\end{array}$} & \multirow{2}{*}{$\begin{array}{l}2014 \\
N= \\
3197\end{array}$} & \multirow{2}{*}{$\begin{array}{l}2015 \\
N= \\
3239\end{array}$} & \multirow{2}{*}{$\begin{array}{l}2016 \\
N= \\
1061\end{array}$} & \multirow{2}{*}{$\begin{array}{l}2017 \\
N= \\
2185\end{array}$} & \multirow{2}{*}{$\begin{array}{l}2018 \\
N= \\
2057\end{array}$} & \multirow{2}{*}{$\begin{array}{l}\text { All } \\
N= \\
21596\end{array}$} & \multirow{2}{*}{$\begin{array}{l}p \text { for } \\
\chi^{2}\end{array}$} & \multirow{2}{*}{$\begin{array}{l}p \text { for } \\
\text { trend }\end{array}$} & \multirow{2}{*}{$\begin{array}{l}\mathrm{OR}(95 \% \\
\mathrm{Cl})\end{array}$} \\
\hline & & & & & & & & & & & & & & \\
\hline \multirow[t]{3}{*}{$\begin{array}{l}\text { Used condoms in the last } \\
\text { commercial sex }\end{array}$} & LC & 91.9 & 91.4 & 96.8 & 95.0 & 98.5 & 98.6 & 98.5 & 98.6 & 98.6 & 96.5 & 0.005 & $\begin{array}{l}< \\
0.001\end{array}$ & $\begin{array}{l}1.36(1.32- \\
1.42)\end{array}$ \\
\hline & $\mathrm{CM}$ & 83.3 & 93.9 & 87.7 & 93.5 & 97.0 & 98.5 & 93.8 & 98.0 & 96.3 & 95.2 & & $<.001$ & $\begin{array}{l}1.24(1.12- \\
1.38)\end{array}$ \\
\hline & All & 91.7 & 91.5 & 96.3 & 94.9 & 98.3 & 98.6 & 97.8 & 98.5 & 98.3 & 96.4 & & $<0.001$ & \\
\hline \multirow{3}{*}{$\begin{array}{l}\text { Consistently used condoms } \\
\text { in commercial sex last } \\
\text { month }\end{array}$} & LC & 67.2 & 69.4 & 87.1 & 81.3 & 85.7 & 95.0 & 92.3 & 92.3 & 94.4 & 85.1 & $<.001$ & $<0.001$ & $\begin{array}{l}1.34(1.32- \\
1.37)\end{array}$ \\
\hline & $\mathrm{CM}$ & 59.5 & 72.7 & 73.9 & 82.6 & 82.8 & 85.1 & 77.0 & 84.3 & 88.8 & 81.7 & & $<0.001$ & $\begin{array}{l}1.12(1.06- \\
1.19)\end{array}$ \\
\hline & All & 67.0 & 69.5 & 86.4 & 81.4 & 85.4 & 94.2 & 90.1 & 91.2 & 93.8 & 84.9 & & $<001$ & \\
\hline \multirow[t]{3}{*}{ History of illicit drugs use } & LC & 2.0 & 0.3 & 0.9 & 1.1 & 1.3 & 0.7 & 1.7 & 1.2 & 2.2 & 1.2 & $\begin{array}{l}< \\
0.001\end{array}$ & 0.007 & $\begin{array}{l}1.08(1.02- \\
1.13)\end{array}$ \\
\hline & $\mathrm{CM}$ & 4.8 & 1.5 & 4.6 & 4.0 & 4.1 & 1.2 & 1.1 & 0.3 & 1.4 & 2.3 & & $\begin{array}{l}< \\
0.001\end{array}$ & $\begin{array}{l}0.77(0.67- \\
0.89)\end{array}$ \\
\hline & All & 2.1 & 0.3 & 1.1 & 1.4 & 1.6 & 0.7 & 1.6 & 1.1 & 2.1 & 1.3 & & 0.082 & \\
\hline \multirow[t]{3}{*}{$\begin{array}{l}\text { Self-reporting STIs in the } \\
\text { last year }\end{array}$} & LC & 3.7 & 1.7 & 3.4 & 5.0 & 4.9 & 2.3 & 2.5 & 5.3 & 4.9 & 3.8 & 0.348 & 0.001 & $\begin{array}{l}1.05(1.02- \\
1.08)\end{array}$ \\
\hline & $\mathrm{CM}$ & 0.0 & 0.0 & 8.5 & 4.7 & 3.0 & 3.8 & 2.2 & 3.3 & 1.4 & 3.3 & & 0.097 & $\begin{array}{l}0.90(0.80- \\
1.02)\end{array}$ \\
\hline & All & 3.6 & 1.6 & 3.7 & 5.0 & 4.7 & 2.4 & 2.5 & 5.0 & 4.6 & 3.7 & & 0.008 & \\
\hline \multirow[t]{3}{*}{$\begin{array}{l}\text { Number of male clients in } \\
\text { the past month }(>60)\end{array}$} & LC & - & - & - & - & - & - & 29.2 & 40.7 & 27.2 & 32.9 & 0.066 & 0.021 & $\begin{array}{l}0.92(0.85- \\
0.99)\end{array}$ \\
\hline & $\mathrm{CM}$ & - & - & - & - & - & - & 23.9 & 33.8 & 29.4 & 29.5 & & 0.186 & $\begin{array}{l}1.15(0.94- \\
1.41)\end{array}$ \\
\hline & All & - & - & - & - & - & - & 28.4 & 39.8 & 27.5 & 32.4 & & 0.116 & \\
\hline \multirow{3}{*}{$\begin{array}{l}\text { Sexing with regular } \\
\text { commercial sexual partners } \\
\text { in the past month }\end{array}$} & LC & - & - & - & - & - & - & 59.4 & 54.9 & 53.6 & 55.5 & $\begin{array}{l}< \\
0.001\end{array}$ & 0.002 & $\begin{array}{l}0.89(0.83- \\
0.96)\end{array}$ \\
\hline & $\mathrm{CM}$ & - & - & - & - & - & - & 87.4 & 61.9 & 46.3 & 65.3 & & $<0.001$ & $\begin{array}{l}0.38(0.30- \\
0.47)\end{array}$ \\
\hline & All & - & - & - & - & - & - & 63.9 & 55.8 & 52.8 & 56.8 & & $\begin{array}{l}< \\
0.001\end{array}$ & \\
\hline \multirow[t]{3}{*}{$\begin{array}{l}\text { Having steady non-paying } \\
\text { sexual partners }\end{array}$} & LC & - & - & - & - & - & - & 16.2 & 11.1 & 11.8 & 12.6 & $\begin{array}{l}< \\
0.001\end{array}$ & 0.001 & $\begin{array}{l}0.84(0.75- \\
0.93)\end{array}$ \\
\hline & $\mathrm{CM}$ & - & - & - & - & - & - & 4.8 & 3.0 & 6.5 & 4.6 & & 0.398 & $\begin{array}{l}1.21(0.78- \\
1.90)\end{array}$ \\
\hline & All & - & - & - & - & - & - & 14.4 & 10.0 & 11.2 & 11.6 & & 0.011 & \\
\hline \multirow[t]{3}{*}{$\begin{array}{l}\text { Age of male clients } \geq 50 \\
\text { years old }\end{array}$} & LC & - & - & - & - & - & - & 27.1 & 19.4 & 24.6 & 23.2 & 0.176 & 0.350 & $\begin{array}{l}0.96(0.88- \\
1.05)\end{array}$ \\
\hline & $\mathrm{CM}$ & - & - & - & - & - & - & 18.7 & 19.1 & 26.2 & 21.0 & & 0.057 & $\begin{array}{l}1.25(0.99- \\
1.57)\end{array}$ \\
\hline & All & - & - & - & - & - & - & 25.8 & 19.3 & 24.8 & 22.9 & & 0.900 & \\
\hline \multirow{3}{*}{$\begin{array}{l}\text { Sexing with commercial } \\
\text { male clients who used } \\
\text { aphrodisiac drugs }\end{array}$} & LC & - & - & - & - & - & - & 8.3 & 9.3 & 7.0 & 8.2 & 0.042 & 0.114 & $\begin{array}{l}0.90(0.79- \\
1.03)\end{array}$ \\
\hline & $\mathrm{CM}$ & - & - & - & - & - & - & 8.3 & 5.4 & 4.7 & 6.1 & & 0.112 & $\begin{array}{l}0.72(0.49- \\
1.08)\end{array}$ \\
\hline & All & - & - & - & - & - & - & 8.3 & 8.8 & 6.8 & 7.9 & & 0.061 & \\
\hline \multirow[t]{2}{*}{$\begin{array}{l}\text { Accurate HIV-/AIDS-related } \\
\text { knowledge }\end{array}$} & LC & 86.5 & 86.9 & 94.6 & 94.2 & 94.6 & 96.1 & 96.0 & 97.8 & 95.1 & 93.7 & $\begin{array}{l}< \\
0.001\end{array}$ & $\begin{array}{l}< \\
0.001\end{array}$ & $\begin{array}{l}1.24(1.21- \\
1.27)\end{array}$ \\
\hline & $\mathrm{CM}$ & 78.6 & 75.8 & 90.8 & 90.9 & 85.8 & 92.0 & 78.5 & 90.3 & 84.6 & 86.8 & & 0.871 & $\begin{array}{l}0.99(0.93- \\
1.06)\end{array}$ \\
\hline
\end{tabular}




\begin{tabular}{|c|c|c|c|c|c|c|c|c|c|c|c|c|c|c|}
\hline \multirow[t]{2}{*}{ Variables } & & 2010 & 2011 & 2012 & 2013 & 2014 & 2015 & 2016 & 2017 & 2018 & All & \multirow{2}{*}{$\begin{array}{l}p \text { for } \\
\chi^{2}\end{array}$} & \multirow{2}{*}{$\begin{array}{l}p \text { for } \\
\text { trend }\end{array}$} & \multirow{2}{*}{$\begin{array}{l}\mathrm{OR}(95 \% \\
\mathrm{Cl})\end{array}$} \\
\hline & & $\begin{array}{l}N= \\
1680\end{array}$ & $\begin{array}{l}N= \\
2366\end{array}$ & $\begin{array}{l}N= \\
2461\end{array}$ & $\begin{array}{l}N= \\
3350\end{array}$ & $\begin{array}{l}N= \\
3197\end{array}$ & $\begin{array}{l}N= \\
3239\end{array}$ & $\begin{array}{l}N= \\
1061\end{array}$ & $\begin{array}{l}N= \\
2185\end{array}$ & $\begin{array}{l}N= \\
2057\end{array}$ & $\begin{array}{l}N= \\
21596\end{array}$ & & & \\
\hline & All & 86.3 & 86.6 & 94.4 & 93.9 & 93.8 & 95.8 & 93.4 & 96.8 & 94.0 & 93.1 & & $\hat{0}_{0.001}^{<}$ & \\
\hline \multirow{3}{*}{$\begin{array}{l}\text { Receiving free condom } \\
\text { distribution programme last } \\
\text { year }\end{array}$} & $\mathrm{LC}$ & 98.7 & 91.1 & 94.9 & 96.5 & 95.2 & 97.2 & 97.2 & 98.9 & 96.6 & 96.1 & $\dot{<}$ & <. & $\begin{array}{l}1.12(1.08- \\
1.15)\end{array}$ \\
\hline & $\mathrm{CM}$ & 97.6 & 92.9 & 95.4 & 89.5 & 86.5 & 90.1 & 80.9 & 97.3 & 87.8 & 89.6 & & 0.351 & $\begin{array}{l}0.97(0.90- \\
1.04)\end{array}$ \\
\hline & All & 98.6 & 91.1 & 94.9 & 95.9 & 94.4 & 96.6 & 94.9 & 98.7 & 95.7 & 95.6 & & $\begin{array}{l}<.001 \\
0.001\end{array}$ & \\
\hline \multirow{3}{*}{$\begin{array}{l}\text { Receiving methadone } \\
\text { maintenance therapy or } \\
\text { needle exchange } \\
\text { programme last year }\end{array}$} & LC & 1.8 & 0.3 & 1.2 & 1.0 & 0.5 & 0.4 & 0.9 & 3.1 & 1.0 & 1.0 & 0.028 & 0.013 & $\begin{array}{l}1.08(1.02- \\
1.14)\end{array}$ \\
\hline & $\mathrm{CM}$ & 4.8 & 0.0 & 4.6 & 4.0 & 2.4 & 0.4 & 0.4 & 0.3 & 0.0 & 1.6 & & $\dot{c}_{0.001}^{<}$ & $\begin{array}{l}0.66(0.55- \\
0.79)\end{array}$ \\
\hline & All & 1.9 & 0.3 & 1.3 & 1.2 & 0.7 & 0.4 & 0.9 & 2.7 & 0.9 & 1.1 & & 0.248 & \\
\hline \multirow[t]{3}{*}{$\begin{array}{l}\text { Receiving peer education } \\
\text { programme last year }\end{array}$} & $\mathrm{LC}$ & 38.9 & 39.9 & 28.8 & 44.5 & 37.3 & 39.9 & 34.9 & 51.6 & 44.5 & 40.0 & \multirow[t]{2}{*}{$\begin{array}{l}< \\
0.001\end{array}$} & <. & $\begin{array}{l}1.05(1.04- \\
1.06)\end{array}$ \\
\hline & $\mathrm{CM}$ & 52.4 & 57.1 & 34.6 & 59.6 & 18.2 & 53.4 & 50.4 & 65.9 & 46.0 & 48.2 & & 0.001 & $\begin{array}{l}1.08(1.03- \\
1.13)\end{array}$ \\
\hline & All & 39.2 & 40.4 & 29.1 & 45.8 & 35.5 & 41.0 & 37.1 & 53.6 & 44.7 & 40.7 & & <. & \\
\hline
\end{tabular}

\section{HIV-/AIDS-related knowledge and intervention services}

As shown in Table 2, during the nine-year period, the awareness rate of HIV/AIDS-related knowledge among LCFSWs was statistically higher than that of CMFSWs $(93.71 \%$ versus $86.76 \%, p<0.001)$, with an increasing trend among LCFSWs $(\mathrm{OR}=1.239,95 \% \mathrm{Cl}: 1.208-1.272)$, while there was no significant change among CMFSWs. A higher proportion of receiving free condom distribution programme could be found among LCFSWs than CMFSWs $(96.12 \%$ versus $89.61 \%, p<0.001)$. However, CMFSWs were more likely to receive methadone maintenance therapy or needle exchange and peer education programmes. There was a significant increase in the utilization of free condom distribution ( $O R=1.116,95 \% \mathrm{Cl}: 1.082-1.151)$, methadone maintenance therapy or needle exchange programmes $(\mathrm{OR}=1.076,95 \% \mathrm{Cl}: 1.015-1.140)$ and peer education programmes $(\mathrm{OR}=1.048,95 \% \mathrm{Cl}: 1.036-1.061)$ among LCFSWs. Increasing trend was also found in peer education programme $(\mathrm{OR}=1.078,95 \% \mathrm{Cl}: 1.031-1.127)$ among CMFSWs, however, the trend of receiving methadone maintenance therapy or needle exchange programme among CMFSWs was decreased ( $\mathrm{OR}=0.657,95 \% \mathrm{Cl}: 0.546-0.790)$.

\section{Risk factors associated with HIV-1 infection}

From 2010 to 2015, as shown in Fig. 2, there were two common risk factors for HIV-1 infection among both LCFSWs and CMFSWs: staying in current residence $>12$ months and self-reporting STIs in the last year. Beyond that, receiveing less than 6 years of education background $(O R=3.731,95 \% \mathrm{Cl}: 1.143-$ 12.185), illicit drugs use ( $\mathrm{OR}=12.398,95 \% \mathrm{Cl}: 6.555-23.449)$,staying in current residence for more than 12 months ( $\mathrm{OR}=1.788,95 \% \mathrm{Cl}: 1.165-2.744)$, and inconsistant condom use in the last month $(\mathrm{OR}=1.595,95 \% \mathrm{Cl}: 1.045-2.436)$ were significantly associated with HIV-1 infection among LCFSWs. For CMFSWs, the unique factor for HIV-1 infection was age $>35$ years old ( $O R=2.428,95 \% \mathrm{Cl}: 1.096-5.381$ ). However, LCFSWs had lower odds to be HIV-1 infected if they had been staying in current residence for $6 \sim 12$ months (OR $=0.397,95 \% \mathrm{Cl}: 0.166-0.952)$.

From 2016 to 2018, self-reporting STIs in the last year was the shared factor significantly related to HIV infection across both groups. For CMFSWs, selfreporting of STIs in the last year ( $\mathrm{OR}=25.936,95 \% \mathrm{Cl}$ : 4.336-155.138), and inconsistent condoms use in commercial sex in the last month $(\mathrm{OR}=3.527,95 \% \mathrm{Cl}$ : 1.153-10.784) were significantly associated with HIV-1 infection, while receiving peer education programme in the past year ( $O R=0.204,95 \%$ Cl: $0.057-$ 0.732) presented a protective effect for HIV-1 infection. Regarding to LCFSWs, self-reporting STIs in the last year (OR $=4.044,95 \% \mathrm{Cl}: 1.558-10.498)$ and engaging in commercial sex with male clients who used aphrodisiac drugs $(\mathrm{OR}=4.029,95 \% \mathrm{Cl}: 1.709-9.497)$ were significantly associated factors for HIV-1 infection.

\section{Risk factors associated with syphilis infection}

From 2010 to 2015, as shown in Fig. 2, staying in current residence for more than 12 months, self-reporting of STIs in the last year and illicit drug use were the shared significant factors to syphilis infection among both LCFSWs and CMFSWs. In addition, inconsistent condoms use in commercial sex in the past month $(\mathrm{OR}=2.506,95 \% \mathrm{Cl}: 1.235-5.086)$ was significant risk factor for syphilis infection among CMFSWs, while low-tier working venues $(\mathrm{OR}=1.882,95 \% \mathrm{Cl}$ : $1.275-$ 2.779), age > 35 years old ( $\mathrm{OR}=1.547,95 \% \mathrm{Cl}: 1.296-1.846)$, and receiving less than 6 or 9 years of education (less than 6 years: $\mathrm{OR}=2.387,95 \% \mathrm{Cl}$ : $1.538-$ 3.705; less than 9 years: $\mathrm{OR}=1.737,95 \% \mathrm{Cl}: 1.30-2.670$ ) were risk factors for syphilis infection among LCFSWs. The marital status of unmarried/divorced/widowed $(\mathrm{OR}=0.835,95 \% \mathrm{Cl}$ : 0.698-0.998) presented a protective effect for syphilis infection among LCFSWs. 
During 2016-2018 period, low-tier working venues, engaging in commercial sex with male clients who used aphrodisiac drugs and self-reporting of STIs in the last year were related to syphilis infection among both LCFSWs and CMFSWs. Regarding to LCFSWs, experience as FSW for more than 5 years (OR $=1.478$, $95 \% \mathrm{Cl}: 1.119-1.951)$, receiving less than 6 years of education $(\mathrm{OR}=1.927,95 \% \mathrm{Cl}$ : $1.008-3.681)$ and history of illicit drug use $(\mathrm{OR}=2.619,95 \% \mathrm{Cl}: 1.084-$ 6.33) were associated with syphilis infection.

\section{Discussion}

The large-scale, long-term consecutive cross-sectional study revealed that the prevalence of HIV-1 (0.91\%) and syphilis infection (5.14\%) in the Sino-Vietnam border areas of Guangxi were similar to those in a previous study (HIV-1: 1.00\%; syphilis: 6.1\%)[9]. The prevalence of syphilis infection among LCFSWs and CMFSWs in this study showed no significant difference. However, higher prevalence of HIV-1 infection was observed among CMFSWs (3.13\%) than LCFSWs $(0.72 \%)[22,23]$. The prevalence of HIV-1 infection among LCFSWs in our study was higher than the national average in FSWs (0.60\%) in China[11] as well as the general population (0.36\%)[24]. Furthermore, we found a downward trend in HIV-1 prevalence among LCFSWs, but this downward trend was not found among CMFSWs with a upward trend from 2016 to 2018, which was of great concerned with us. The higher HIV-1 prevalence among CMFSWs than LCFSWs was consistent with previous studies[15, 17]. It demonstrated that CMFSWs in China were typically impoverished, facing language barriers and lacking of Chinese legal protection, all of which may prevent them from effectively participating in local HIV-1 intervention programmes[15], thus putting them at higher risk of HIV-1 infection.

In this study, there were several differences in demographic characteristics and sexual behaviours between LCFSWs and CMFSWs, which may account for the difference in HIV-1 prevalence between the two groups. For example, our results showed that CMFSWs were more likely to be unmarried/divorced/widowed, reporting commercial sexual debut at age $<18$ years old, working in low-tier venues and staying in current residence for less than 6 months than LCFSWs. Previous study has found that women who were divorced or widowed usually face greater financial pressure to support their families and were willingly to engage in unprotected sex for more money[9], which increases their risk of HIV-1 infection. In addition, engaging in sex work early could affect risky behaviours in adulthood, and such FSWs are unlikely to request condoms use on their own initiative[25]. Inconsistency in condoms use was common in CMFSWs from low-tier working venues who had insufficient HIV/AIDS-related knowledge[15]. High mobility in CMFSWs was independently associated with HIV-1 infection[22].

CMFSWs were more likely to use illicit drugs than LCFSWs. Aside from the risk of HIV-1 infection posed by drug use alone, drug-taking FSWs may be at greater risk for sexual HIV-1 acquisition. It has been confirmed that drug addicted FSWs were more likely to have unprotected sex[26], thus significantly increasing the risks of both new and established HIV-1 infections[27] as well as HCV infection[15]. Drug addicted FSWs often trade sex for drugs and are heavily influenced by drugs. They usually reported larger number of clients or more frequent sex, and their ability to negotiate safe sex was impaired while under the influence of drugs[28]. It was also possible that drug addicted FSWs have more risky sexual partners such as male clients who inject drugs[29, 30]. The relatively high HIV1 prevalence and increasing unsafe behaviours in CMFSWs highlight the importance of governmental intervention in this neglected subgroup to prevent the spread of HIV/STIs from high-risk groups to the general population, especially male clients of FSWs.

Statistically significant difference was found between LCFSWs and CMFSWs in terms of the proportion of participants receiving free condom distribution programme in the past year, with the former having a higher proportion and increasing year by year. Therefore, the accuracy of HIV/AIDS-related knowledge was improved in LCFSWs, but not in CMFSWs. It is known that knowledge about HIV/AIDS significantly affects FSWs' ability to perceive risk and engage in safe behaviours accordingly. In particular, inadequate knowledge of HIV/AIDS directly contributes to the failure of condom use and other preventive measures[31]. In accordance with previous study, self-reported risk behaviours, especially inconsistent condom use, are significantly reduced among Chinese FSWs[32]. Although this trend also exists among CMFSWs, the percentage of inconsistent condom use among CMFSWs is still significantly higher than that of their Chinese counterparts. FSWs often compromise on condom use in order to earn more money or establish a closer long-term relationship with their clients[33]. Behavioural intervention has been shown to be effective in improving condom use[34], which suggests that we need to improve the behavioural intervention support system targeting CMFSWs and strengthen the bilateral cooperation between China and Vietnam.

The logistic regression results confirmed that history of illicit drugs use was a risk factor for HIV-1 and syphilis infection in LCFSWs in $2010-2015$ period, but it was not a risk factor in 2016-2018 period, which was in consistent with the trend of declining drug use behavior among Chinese FSWs[32]. In addition,selfreporting STIs in the last year and staying in current residence for more than 12 months were significantly associated with HIV-1 infection in both LCFSWs and CMFSWs, while staying in current residence for more than 12 months were related to syphilis infection among CMFSWs and LCFSWs, respectively. A metaanalysis reported that STIs as a superior role for contributing to HIV infection[35]. Therefore, there is no doubt that self-reporting STIs has been a associatted factor to HIV infection among FSWs in a decade. A relatively long period of staying in the same residence was also associated with HIV-1 and syphilis infection, which could be explained by the fact that time spent engaging in commercial sex is a driver of vulnerability to HIV-1 and syphilis infection in FSWs[26, 27, 36].

During 2010-2015, self-reporting of STIs in the last year had a 2.7-fold and 3.9-fold increase of HIV-1 infection among both LCFSWs and CMFSWs. This effect had risen to 25.9-fold among CMFSWs in 2016-2018 period. The presence of STIs could facilitate the acquisition and transmission of HIV-1 through sexual contact[37, 38]. It is worth noting that self-reporting of STIs was also correlated to syphilis infection among both LCFSWs and CMFSWs in two study periods. Since syphilis infection can facilitate HIV-1 transmission by increasing susceptibility to HIV-1 infection, the co-infection of HIV-1 and syphilis has been commonly observed[9]. This phenomenon may be due to their shared transmission routes, co-risk factors and adverse interaction[39]. The significant association between HIV-1 and STIs as well as the increased prevalence of syphilis infection in Guangxi indicated the increasing role of commercial sexual transmission in China's HIV/AIDS epidemic. 
During 2016-2018, to investigate whether demographic and sexual behavior characteristics of male clients or sexual partners of FSWs would influence their HIV-1 and syphilis infection, we added questions related to male clients or sexual partners in the survey. For CMFSWs, self-reporting STIs in the last year and inconsistent condom use in the last month were the associated factors with HIV-1 infection, reflecting the significant impact of sexual behaviour and STIs on access to HIV-1. While for LCFSWs, women who were self-reporting STIs in the last year and sexing with male clients who use aphrodisiac drugs were found more likely to be HIV-1 infected. As known, erectile dysfunction is the most common type of sexual dysfunction and is most prevalent among elderly men, causing a variety of biological and psychological responses[40]. Aphrodisiacs can significantly improve erectile dysfunction, and are widely used by male clients in Guangxi due to its low price and high availability[19]. Male clients who used aphrodisiac drugs were more likely to have non-steady sexual partners[41], leading to the potential rapid spread of HIV-1.

In this study, peer education was a strong protective factor for HIV-1 infection among CMFSWs. Peer education has become a global health promotion strategy for HIV-1 infection worldwide and has been shown to be an effective tool with long-tern impact for reducing HIV-1 prevalence among all high-risk groups, by promoting HIV-1 testing and condom use while reducing equipment sharing and unprotected sex[42]. Previous studies of FSWs have shown that peer-assisted multicomponent behavioural interventions were beneficial to reductions in unprotected sex with a stable partner, and the moderate initial effect of the intervention was not only sustained but actually strengthened over time[43, 44], providing an idea for joint intervention measures based on peer education. Thus emphasizing the importance of peer education for CMFSWs and reminding the government departments to pay more attention to peer education and peer-assisted behavioural interventions targeting this vulnerable subgroup.

The strengths of this study include a large sample size, longer and continuous observation time (9 years), and the detail behavioral and sociological factors associated with HIV-1 infection. However, being an observational study, our results is subject to several limitations. First, the cumulative data were based on annual surveys implemented in the same cities from 2010 to 2018. It is likely that some FSWs participated in the survey more than once. However, the specific monitoring sites in this study varied from year to year, and less than $10 \%$ of all FSWs repeated participation in the survey, as they were highly mobile. We estimated that the percentage of overlap, if present, was very small and had no significant effects. Second, since commercial sex work is illegal in China, it was impossible to employ random sampling to recruit participants in this study, likely affecting the representative of the samples. However, the large sample size and multicentre design potentially reduced the selection bias. Third, some outcomes of CMFSWs, such as HIV- 1 and syphilis infections, self-reporting of STIs and history of illicit drug use, may have predated their migration to China. Therefore, the relationship between HIV-1 infection and potential risk factors, as well as the true HIV-1 burden among FSWs in Guangxi, may be overestimated.

\section{Conclusions}

In conclusion, the higher prevalence of HIV-1 infection and frequent risk behaviours especially the inconsistent condoms use found among CMFSWs in Guangxi, indicating that the current preventive intervention programs in China are not well targeted at this high-risk subgroup. The sexual behaviour characteristics of male clients could influence the infection of HIV-1 and syphilis in FSWs. In order to achieve the 90-90-90 goals early and end the HIV/AIDS epidemic in border areas, more effective and comprehensive intervention strategies need to be developed, and more attention should be paid to improving the effectiveness of interventions.

\section{List Of Abbreviations}

\begin{tabular}{|ll|}
\hline HIV-1 & Human Immunodeficiency Virus type 1 \\
\hline HCV & Hepatitis C Virus \\
\hline AIDS & Acquired Immune Deficiency Syndrome \\
\hline FSWs & Female Sex Workers \\
\hline LCFSWs & Local Chinese Female Sex Workers \\
\hline CMFSWs & Cross-border Migrant Female Sex Workers \\
\hline STIs & Sexually Transmitted Infections \\
\hline CDC & Center for Disease Control and Prevention \\
\hline ELISA & Enzyme-linked immunosorbent assay \\
\hline WB & Western Blot \\
\hline RPR & Rapid Plasma Reagin \\
\hline TPPA & Treponema Pallidum Particle Agglutination \\
\hline ORs & Odd Ratios \\
\hline Cls & Confidence Intervals \\
\hline
\end{tabular}

\section{Declarations}

Ethics approval and consent to participate 
This study was reviewed and approved by the Guangxi Institutional Review Board (GXIRB2014-0041). Written informed consent was obtained from the participants.

\section{Consent for publication}

Not applicable.

\section{Availability of data and materials}

The datasets used and analysed during the current study are available from the corresponding author on reasonable request.

\section{Competing interests}

The authors declare that they have no competing interests.

\section{Funding}

The study was supported by National Key Science and Technology Project of China (Grant No. 2018ZX10101002-001-006), National Natural Science Foundation of China (Grant No. 82060610), Guangxi Scientific and Technological Key Project (Gui Ke AB18050022), Thousands of Young and Middle-aged Key Teachers Training Program in Guangxi Colleges and Universities (To Junjun Jiang), and Guangxi Bagui Scholar (To Junjun Jiang).

\section{Authors' contributions}

$\mathrm{BL}, \mathrm{FZ}$ and DW collaborated in the writing of the manuscript. PZ, GW, SM and DV involved in the design and conduction of the survey. AN, YO and ZW performed the statistical analyses. $\mathrm{HX}, \mathrm{XL}, \mathrm{JL}, \mathrm{YL}$ and $\mathrm{NV}$ assisted with data management and analysis. JH and JJ complemented the manuscript with contextual data. DL, HL and LY chose the main directions for data analysis and participated in the interpretation of the results. All authors contributed to the revision of the manuscript before submission and approved the final version.

\section{Acknowledgements}

The authors thank the health workers in Chongzuo CDC, Fangchenggang CDC and Baise CDC for their hard work in carrying out the surveys and data collection, as well as all the participants for their voluntary cooperation in the survey.

\section{References}

1. Wang HB, Yang Z, Zhu H, Mo QH, Tan H. High HIV-1 prevalence and viral diversity among entry-exit populations at frontier ports of China, 2012-2016: A cross-sectional molecular epidemiology study. Infection Genetics and Evolution. 2018;65:231-7.

2. Pang X, Wei H, Huang J, He Q, Tang K, Fang N, et al. Patterns and risk of HIV-1 transmission network among men who have sex with men in Guangxi, China. Sci Rep. 2021;11(1):513.

3. Chen H, Luo L, Pan SW, Lan G, Zhu Q, Li J, et al. HIV Epidemiology and Prevention in Southwestern China: Trends from 1996-2017. Curr HIV Res. 2019;17(2):85-93.

4. Lu L, Jia M, Ma Y, Yang L, Chen Z, Ho DD, et al. The changing face of HIV in China. Nature. 2008;455(7213):609-11.

5. WHO. Report on global sexually transmitted infection surveillance. 2018.

6. Su S, Chow EP, Muessig KE, Yuan L, Tucker JD, Zhang X, et al. Sustained high prevalence of viral hepatitis and sexually transmissible infections among female sex workers in China: a systematic review and meta-analysis. BMC Infect Dis. 2016;16:2.

7. Zhu J, Yuan R, Hu D, Zhu Z, Wang N, Wang B. HIV prevalence and correlated factors of female sex workers and male clients in a border region of Yunnan Province, China. Int J STD AIDS. 2018;29(5):424-34.

8. Wang L, Tang WM, Wang L, Qian SS, Li YG, Xing JN, et al. The HIV, Syphilis, and HCV Epidemics Among Female Sex Workers in China: Results From a Serial Cross-Sectional Study Between 2008 and 2012. Clinical Infectious Diseases. 2014;59(1):E1-E9.

9. Zhou Y, Li X, Zhang C, Tan G, Stanton B, Zhang X, et al. Rates of HIV, syphilis, and HCV infections among different demographic groups of female sex workers in Guangxi China: evidence from 2010 national sentinel surveillance data. AIDS Care. 2013;25(11):1433-41.

10. Chen Y, Shen Z, Morano JP, Khoshnood K, Wu Z, Lan G, et al. Bridging the epidemic: a comprehensive analysis of prevalence and correlates of HIV, hepatitis C, and syphilis, and infection among female sex workers in Guangxi Province, China. PLoS One. 2015;10(2):e0115311.

11. Poon AN, Li Z, Wang N, Hong Y. Review of HIV and other sexually transmitted infections among female sex workers in China. AIDS Care. 2011;23 Suppl 1:5-25.

12. Chen XS, Wang QQ, Yin YP, Liang GJ, Jiang N, Yang LG, et al. Prevalence of syphilis infection in different tiers of female sex workers in China: implications for surveillance and interventions. BMC Infect Dis. 2012;12:84.

13. Zhu BY, Bu J, Huang PY, Zhou ZG, Yin YP, Chen XS, et al. Epidemiology of sexually transmitted infections, HIV, and related high-risk behaviors among female sex workers in Guangxi Autonomous Region, China. Jpn J Infect Dis. 2012;65(1):75-8.

14. Chen Y LW, Ted. Hammett, Meng DH, Xiang SM. Investigation and analysis of AIDS related knowledge, behavior and HIV infection among female sex workers in border counties of Guangxi. Chinese Journal of Disease Control \& Prevention. 2010;14(7):619-22. 
15. Yu YJ, Li X, Tam CC, Zhou Y, Chen Y, Shen Z. Demographic and behavioral correlates of HIV/STI among Vietnamese female sex workers in southwest China. AIDS Care. 2016;28(11):1455-60.

16. Thuong NV, Nhung VT, Nghia KV, Tram LT, O'Farrell N. HIV in female sex workers in five border provinces of Vietnam. Sex Transm Infect. 2005;81(6):477-9.

17. Wang J, Ding G, Zhu Z, Zhou C, Wang N. Analysis of HIV Correlated Factors in Chinese and Vietnamese Female Sex Workers in Hekou, Yunnan Province, a Chinese Border Region. PLoS One. 2015;10(6):e0129430.

18. Chen L, His JH, Wu X, Shen Z, Lu H, Chen H, et al. Disparities in HIV and syphilis prevalence and risk factors between older male clients with and without steady sex partners in southwestern rural China. BMC Infect Dis. 2017;17(1):269.

19. Tang ZZ, Wu XH, Li GJ, Shen ZY, Zhang HM, Lan GH, et al. Aphrodisiac Use Associated with HIV Infection in Elderly Male Clients of Low-Cost Commercial Sex Venues in Guangxi, China: A Matched Case-Control Study. Plos One. 2014;9(10).

20. Lai JZ, Qin CW, Nehl EJ, Jiang JJ, Huang YX, Liang BY, et al. HIV prevalence among female sex workers in Guigang City, Guangxi, China: an 8-year consecutive cross-sectional study. Bmc Public Health. 2018;18.

21. The State Council AIDS Working Committee Office tPsRoC. China HIV/AIDS monitoring and evaluation framework (trial). Beijing: Beijing medical publishing house. 2007.

22. Yu J, Nehl EJ, Dinh VP, Liang B, Son NV, Meng D, et al. Vietnamese female sex workers in rural cross-border areas of Guangxi, China: migration and HIV/STI risk behaviors. AIDS Care. 2020;32(12):1515-23.

23. Zhang Y, Liang B, Liu D, Wei G, Mo S, Nong A, et al. Migrant female sex workers working at the Sino-Vietnamese border for a short time have a higher risk of HIV transmission: a consecutive cross-sectional study. AIDS Res Ther. 2020;17(1):4.

24. Zhang L, Chow EP, Jing J, Zhuang X, Li X, He M, et al. HIV prevalence in China: integration of surveillance data and a systematic review. Lancet Infect Dis. 2013;13(11):955-63.

25. Parcesepe AM, L'Engle KL, Martin SL, Green S, Suchindran C, Mwarogo P. Early sex work initiation and condom use among alcohol-using female sex workers in Mombasa, Kenya: a cross-sectional analysis. Sexually Transmitted Infections. 2016;92(8):593-8.

26. Szwarcwald CL, Damacena GN, de Souza PRB, Guimaraes MDC, de Almeida WD, Ferreira APD, et al. Factors associated with HIV infection among female sex workers in Brazil. Medicine. 2018;97:S54-S61.

27. Sopheab H, Chhea C, Tuot S, Muir JA. HIV prevalence, related risk behaviors, and correlates of HIV infection among people who use drugs in Cambodia. Bmc Infectious Diseases. 2018;18.

28. Xu J, Smith MK, Ding G, Chu J, Wang H, Li Q, et al. Drug use and sex work: competing risk factors for newly acquired HIV in Yunnan, China. PLoS One. 2013;8(3):e59050

29. Reilly KH, Wang J, Zhu Z, Li S, Yang T, Ding G, et al. HIV and associated risk factors among male clients of female sex workers in a Chinese border region. Sex Transm Dis. 2012;39(10):750-5.

30. Hail-Jares K, Choi S, Duo L, Luo Z, Huang ZJ. Occupational and demographic factors associated with drug use among female sex workers at the ChinaMyanmar border. Drug Alcohol Depend. 2016;161:42-9.

31. Wong WCW, Wang YL. A qualitative study on HIV risk behaviors and medical needs of sex workers in a China/Myanmar border town. Aids Patient Care and Stds. 2003;17(8):417-22.

32. Chow EP, Muessig KE, Yuan L, Wang Y, Zhang X, Zhao R, et al. Risk behaviours among female sex workers in China: a systematic review and data synthesis. PLoS One. 2015;10(3):e0120595.

33. Ma Q, Jiang J, Pan X, Cai G, Wang H, Zhou X, et al. Consistent condom use and its correlates among female sex workers at hair salons: a cross-sectional study in Zhejiang province, China. BMC Public Health. 2017;17(1):910.

34. Chow EP, Tung K, Tucker JD, Muessig KE, Su S, Zhang X, et al. Behavioral Interventions Improve Condom Use and HIV Testing Uptake Among Female Sex Workers in China: A Systematic Review and Meta-Analysis. AIDS Patient Care STDS. 2015;29(8):454-60.

35. Kabapy AF, Shatat HZ, Abd El-Wahab EW. Attributes of HIV infection over decades (1982-2018): A systematic review and meta-analysis. Transbound Emerg Dis. 2020;67(6):2372-88.

36. Bowring AL, Ketende S, Billong SC, Mfochive Njindam I, Rao A, Decker MR, et al. Characterizing Sociostructural Associations With New HIV Diagnoses Among Female Sex Workers in Cameroon. J Acquir Immune Defic Syndr. 2019;80(3):e64-e73.

37. Ruan Y, Cao X, Qian HZ, Zhang L, Qin G, Jiang Z, et al. Syphilis among female sex workers in southwestern China: potential for HIV transmission. Sex Transm Dis. 2006;33(12):719-23.

38. Galvin SR, Cohen MS. The role of sexually transmitted diseases in HIV transmission. Nat Rev Microbiol. 2004;2(1):33-42.

39. Shimelis T, Lemma K, Ambachew H, Tadesse E. Syphilis among people with HIV infection in southern Ethiopia: sero-prevalence and risk factors. BMC Infect Dis. 2015;15:189.

40. Chen L, Shi GR, Huang DD, Li Y, Ma CC, Shi M, et al. Male sexual dysfunction: A review of literature on its pathological mechanisms, potential risk factors, and herbal drug intervention. Biomed Pharmacother. 2019;112:108585.

41. Chen L, His JH, Wu XH, Shen ZY, Lu HX, Chen HH, et al. Disparities in HIV and syphilis prevalence and risk factors between older male clients with and without steady sex partners in southwestern rural China. Bmc Infectious Diseases. 2017;17.

42. He J, Wang Y, Du Z, Liao J, He N, Hao Y. Peer education for HIV prevention among high-risk groups: a systematic review and meta-analysis. BMC Infect Dis. 2020;20(1):338. 
43. Yang X, Xia G, Li X, Latkin C, Celentano D. The efficacy of a peer-assisted multi-component behavioral intervention among female entertainment workers in China: an initial assessment. AIDS Care. 2011;23(11):1509-18.

44. Yang X, Xia G. Effectiveness of a Peer-Assisted Multicomponent Behavioral Intervention in HIV Risk Reduction Among Female Entertainment Workers in China. AIDS Educ Prev. 2015;27(5):446-64.

\section{Figures}
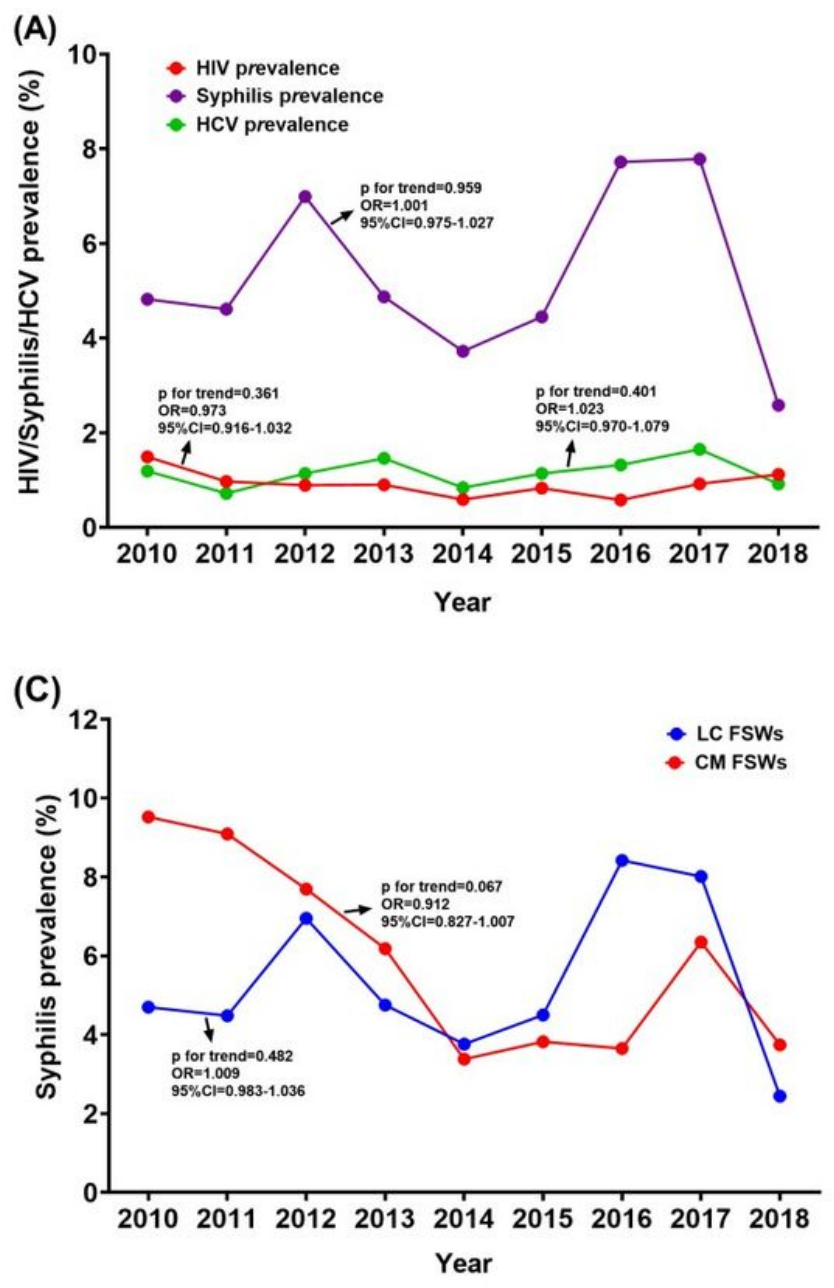
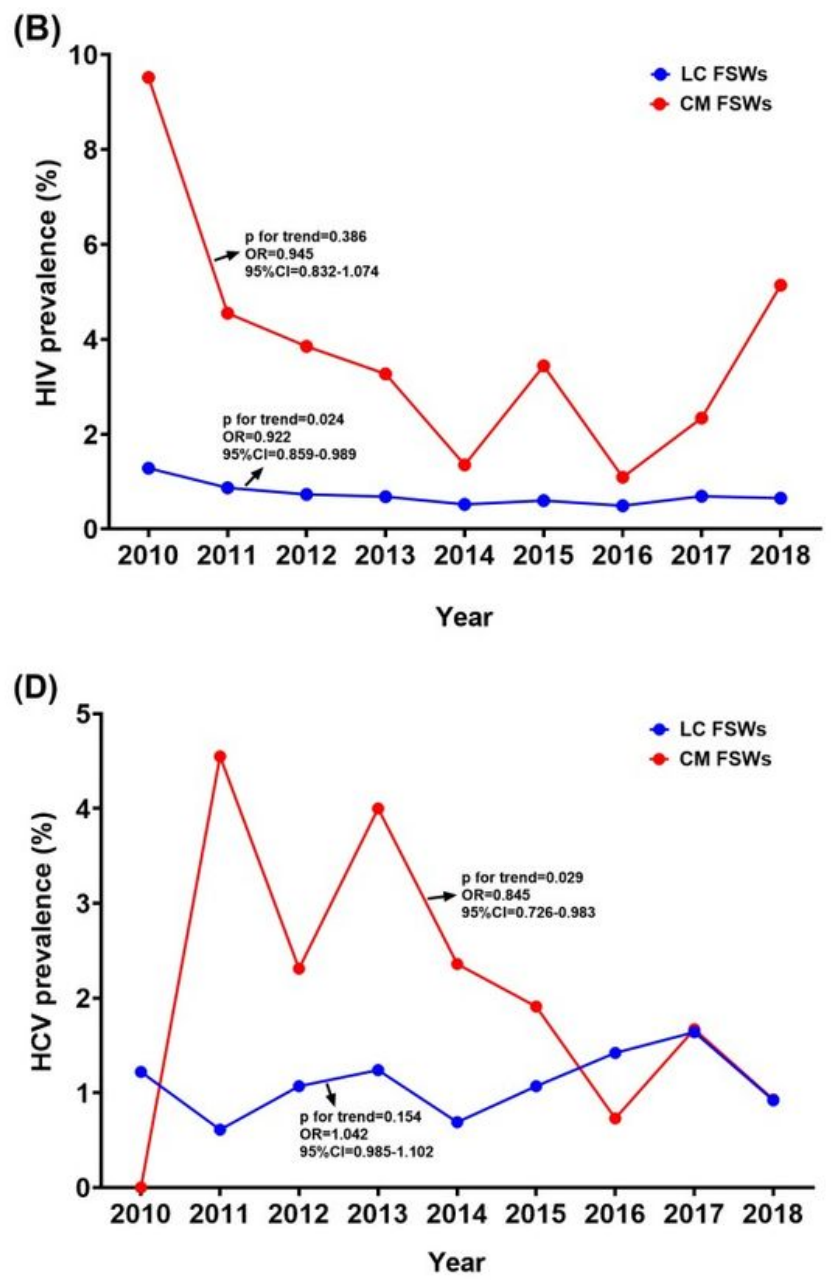

\section{Figure 1}

Prevalence of HIV, syphilis and HCV among local Chinese and cross-border migrant FSWs from 2010 to 2018. (A) The overall prevalence of HIV, syphilis and HCV. (B) Prevalence of HIV infection. (C) Prevalence of syphilis infection. (D) Prevalence of HCV infection. Abbreviation: CMFSWs, cross-border migrant female sex workers; LCFSWs, local Chinese female sex workers. 
(A)

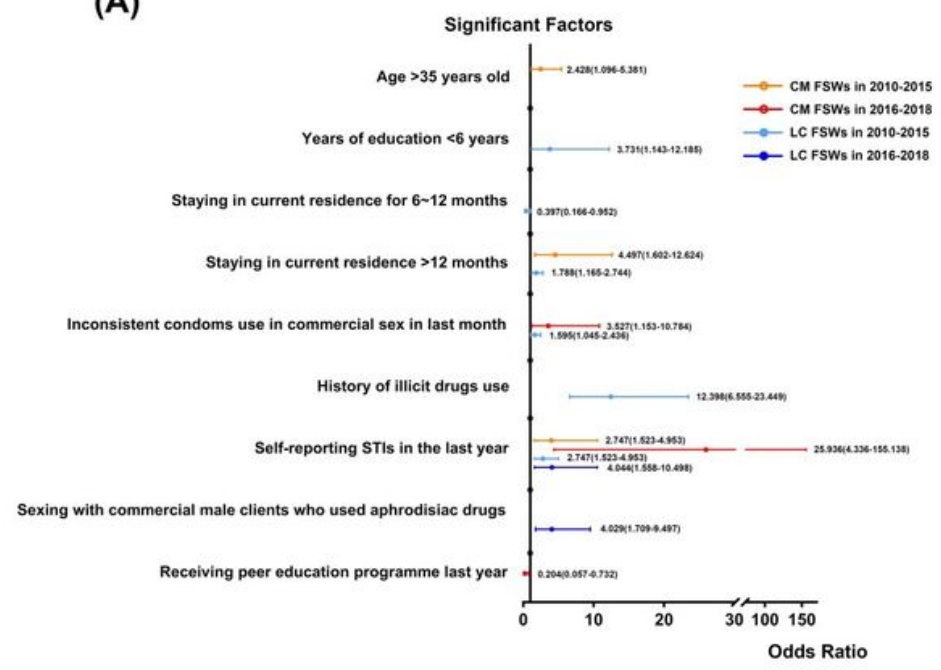

(B)

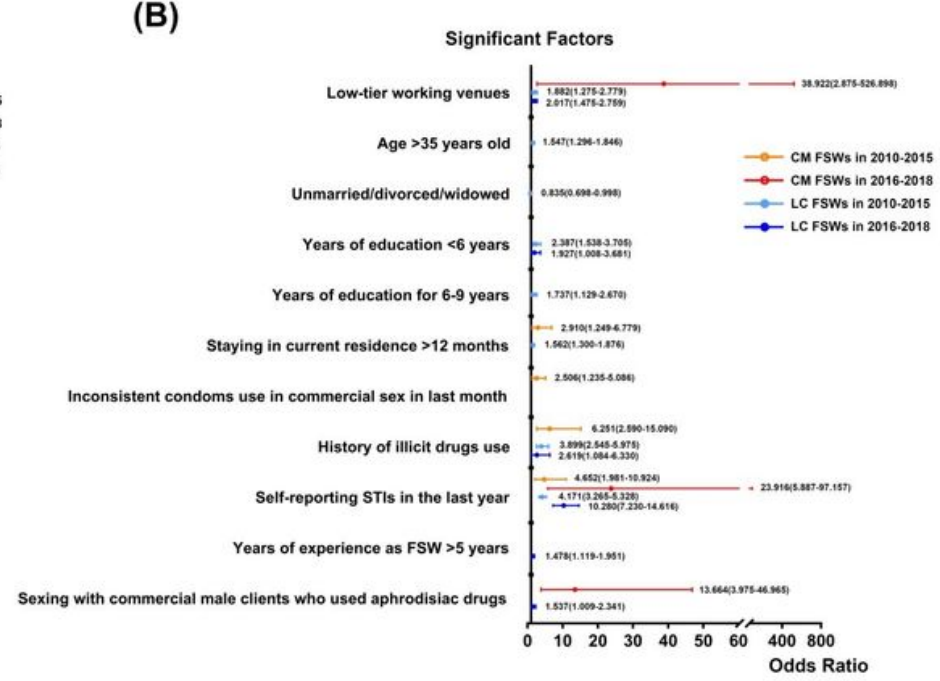

Figure 2

Associated factors of HIV and syphilis infection among local Chinese and cross-border migrant FSWs during 2010-2015 and 2016-2018. (A) Associated factors of HIV infection. (B) Associated factors of syphilis infection. Abbreviations: CMFSWs, cross-border migrant female sex workers; LCFSWs, local Chinese female sex workers.

\section{Supplementary Files}

This is a list of supplementary files associated with this preprint. Click to download.

- supplementarymaterials.docx 